\title{
KIVA Program for Trauma Healing due to Cyber-bullying among Students
}

\author{
Aris Widiyanto, Joko Tri Atmojo, Rina Tri Handayani
}

School of Health Sciences Mamba'ul 'Ulum, Surakarta

\begin{abstract}
Background: Cyber-bullying involves the use of electronic media with the aim of causing damage, humiliation, suffering, fear, and despair for individuals who are the targets of aggression. The impact of cyber-bullying is not only affect their daily lives but may continue to adulthood. KiVa is research-based anti-bullying program that has been developed in the University of Turku, Finland, with funding from the Ministry of Education and Culture. The effectiveness of KiVa has been shown in a large randomized controlled trial. This study aimed to describe the health problems and evidence-based intervention programs that support trauma healing through KiVa program.

Subjects and Method: A systematic review was conducted by searching the following databases: PubMed, SAGE Journals and Virtual Health Library from 2010 until 2018. The keywords for this review included cyber-bullying AND children, cyber-bullying AND mental health AND Children, cyber-bullying in children AND intervention. The inclusion criteria were systematic review, clinical review, cohort study, cross-sectional study, clinical study and guidelines. After the review process, 19 articles and 1 RCT were included.

Results: Internationally, the impact of cyber-bullying on children has been linked to poor mental health including overall psychological health, low self-esteem, depression and anxiety and violent behavior. Another study showed that bully victims had the highest risk of self-injury, suicidal ideation, suicide plan and effort. KiVa is a school-based anti-bullying program from Finland involving teachers, students and parents. The KiVa principle is the prevention of bullying by cultivating character in students by forming group norms. The forms vary, can be videos, online games, surveys, posters, and guidebooks for parents. Children are taught a sense of responsibility and empathy. Previous RCT studies which involved 2,042 students in grades 4 and 6 showed that KiVa reduced intimidation, pro-intimidation, and victimization. And increasing attitude and empathy for victims. Overall, the findings provide evidence of the effectiveness of the KiVa Program.
\end{abstract}

Conclusion: Cyber-bullying is associated with emotional stress and even suicide attempts. Parents, health personnels, and educators must be aware of the long-term risks so that prevention and treatment programs such as KiVa should be considered to be applied in Indonesia.

Keywords: Cyber-bullying, children, mental health

\section{Correspondence:}

Aris Widiyanto. School of Health Sciences Mamba'ul 'Ulum, Surakarta, Jl. Ring Road Utara, Tawangsari, Mojosongo, Jebres, Surakarta, Central Java. Email: widiyantoaris99@gmail.com. Mobile: +6281329209095

\begin{tabular}{l}
\hline BACKGROUND \\
\hline Bullying is a phenomenon that becomes a \\
factor of depression and anxiety in adoles- \\
cents (Arseneault, 2017). Bullying, defined \\
as a dangerous aggressive action that is \\
repeated and directed at someone or \\
colleagues who are less strong or weaker \\
mentally (Araya et al., 2017). Bullying is
\end{tabular}

very common in schools throughout the world.

A large study which involved 40 countries in 2009 showed that $10.7 \%$ of students were intimidated by others on a regular basis, $12.6 \%$ stated that they were repeatedly harassed by their peers, and $3.6 \%$ were reported as bullies and victims 
of intimidation (Craig et al., 2009). Adolescent Health and Welfare Survey in Australia shows that $31 \%$ of experienced teens experience verbal intimidation, $11 \%$ physical intimidation and 14\% social intimidation (Thomas et al., 2016).

Cyberbullying is a new form of intimidation that has been defined as "destructive aggressive and repeated actions through the use of computers, telephones, and other electronic devices". Cyberbullying involves the use of electronic media with the aim of causing damage, humiliation, suffering, fear and despair for individuals who are the targets of aggression. Criteria for the act of "cyberbullying" include: attacks must be intentional, occur repeatedly and cause psychological distress (Hutson et al., 2018).

In addition, the impact of cyberbullying does not only occur in everyday life but can continue until adolescence. Research has found that cyberbullying can cause symptoms of depression, anxiety, selfesteem problems, academic problems, drug use, physical health problems and have a stronger relationship with suicidal ideas (Van Geel et al., 2014; Giumetti and Kowalski, 2015; Hutson et al., 2018).

Various effective school-based suppression prevention programs are developed in order to avoid children from disturbances and also the behavior of bullying and cyberbullying. According to a meta-analysis by Ttofi and Farrington (2011) one of the programs called KiVa can reduce the prevalence of bullies and victims by $17-23 \%$.

KiVa is a research-based anti-bullying program that has been developed at Turku University, Finland, and the funding is from the KiVa Ministry of Education and Culture and shown in large randomized controlled trials. This study aims to describe the health problems and evidence- based intervention programs that support trauma healing through the KiVa program.

\section{SUBJECTS AND METHOD}

Systematic reviews were conducted by searching the following databases: PubMed, SAGE Journal, and Virtual Health Library from 2010 to 2018. Systematic database searches are conducted from March 30 to December 20, 2018.

Keywords for this review include cyberbullying and children, cyberbullying and mental health and children, cyberbullying in children and intervention.

\section{Inclusion criteria}

Inclusion criteria are systematic reviews, clinical reviews, cohort studies, cross-sectional studies, clinical studies and program guidelines.

\section{RESULTS}

The article selection process was based on the Selected Reporting Items for Systematic Reviews and the Preferred Reporting Items for Systematic Reviews and Meta-Analyzes (PRISMA). The initial selection of articles resulted in 751 articles with the 2010-2018 of publication time.

After removing duplicates and applying exclusion criteria, 140 articles were analyzed further. After carefully examining the full text article, finally 19 articles were included for further discussion which involved 1 clinical trial article with an RCT design, a search summary can be seen in (Figure 1).

\section{Definition of Cyberbullying}

The term "cyberbullying" was first created in 1999, there was no general consensus on its definition (Schneider et al., 2012).

Patchin and Hinduja (2006) defined cyberbullying as "intentional and repeated losses incurred through the use of computers, telephones, or other electronic devices. Kowalski et al., (2014) defined it as "The 
use of electronic communication technology to bully or intimidate others".

Studies have shown that cyber-bullying can cause damage more than ordinary

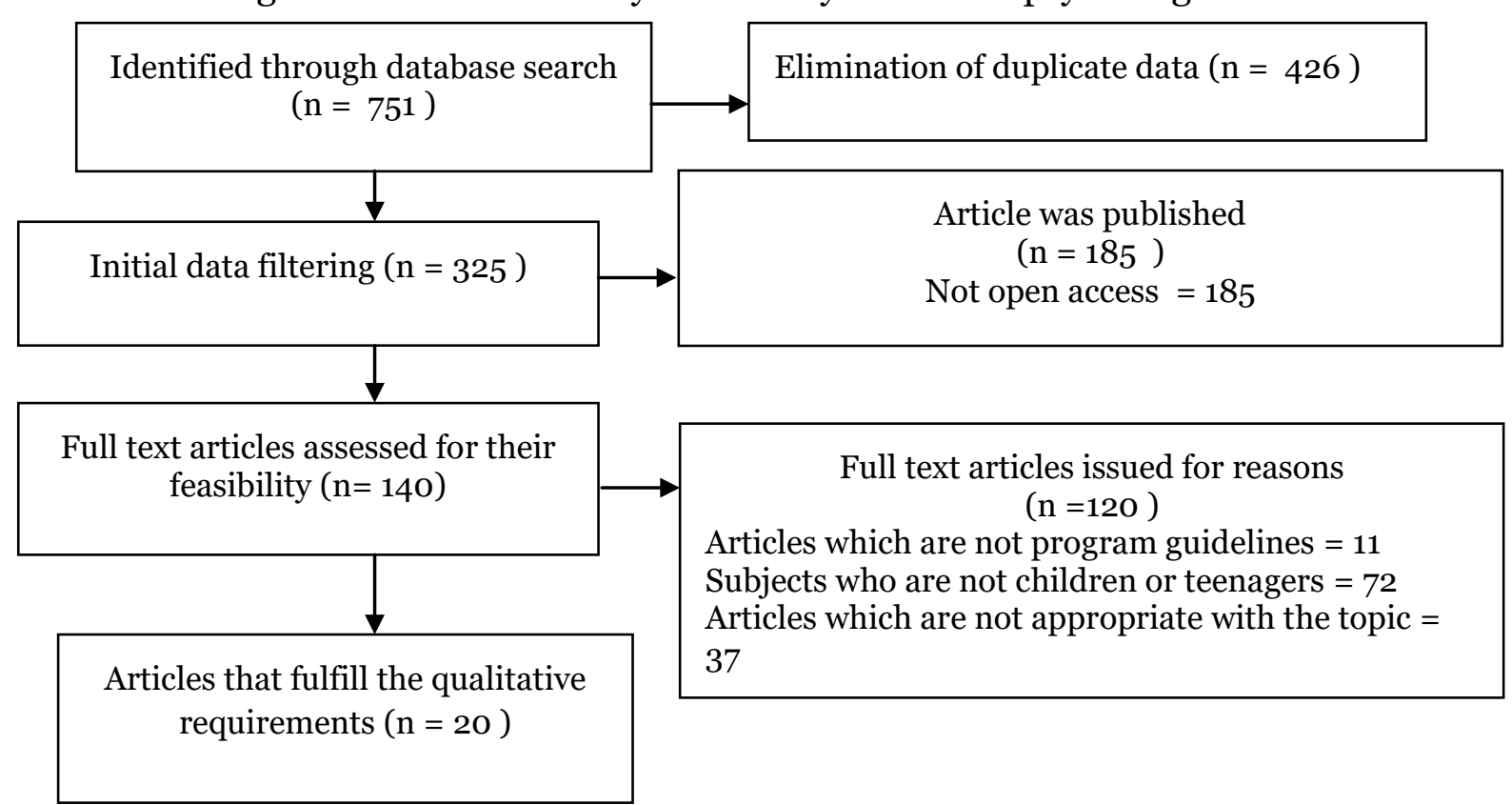

bullying (Donnerstein et al., 2017). Briefly, the criteria for "cyber-bullying" were: attacks must be intentional, occur repeatedly and cause psychological distress.
Article was published

$(n=185)$

Not open access $=185$

$$
\text { (n) iss }
$$

Articles which are not program guidelines $=11$

Subjects who are not children or teenagers $=72$

Articles which are not appropriate with the topic $=$

\section{Figure 1. Research plot}

\section{Cyberbullying imp act on physical and mental health}

The impact of cyberbullying especially on mental and physical health did not only have an impact on daily life but can continue to adulthood.

The following are some of the impacts according to various studies:

a. Albdour et al. (2019) reported that there was a positive correlation between physical complaints $(\mathrm{r}=0.40 ; \mathrm{p}<0.001)$ and psychological pressure $(\mathrm{r}=0.33 ; \mathrm{p}$ $<0.001)$ on cyber-bullying.

b. Gini and Espelage (2014) reported that adolescents involved in cyber-bullying showed an increased risk of emotional problems, difficulty in interacting, feelings of insecurity, psychological problems such as fear, helplessness, loss of self-confidence, and sadness.

c. Schneider et al. (2012) stated that cyberbullying decreased school grades, stress, anxiety, and depression. d. Låftman et al. (2013); Van Geel et al. (2014) stated that victims of cyberbullying were more likely to think about suicide.

Internationally, the impact of cyberbullying on children has been linked to poor mental health including overall psychological health, low self-esteem, depression and anxiety and violent behavior. In another study, it showed that bully victims had the highest risk of self-injury ( $\mathrm{PR}=4.7$; $95 \% \mathrm{CI}=3.26$ to 6.83 ), suicidal ideas ( $\mathrm{PR}=$ 4.3 ; $95 \% \mathrm{CI}=2.83$ to 6.49 ), suicide plans $(\mathrm{PR}=4.1 ; 95 \% \mathrm{CI}=2.54$ to 6.58$)$, and suicide attempts $(\mathrm{PR}=2.7 ; 95 \% \mathrm{CI}=1.39$ to 5.13 ).

\section{Recommended anamnesa and questions}

According to Donnerstein et al., (2017), here are some recommendations for anamnesis: 
a. Ask the patients to explain their negative and positive experiences about the use of digital technology

b. Ask the patients if they have seen other people who experiences cyber-bullying before. If yes, what kind of problems do they have and what do they think about the problem?

c. Ask the patients to describe the type of social media application that causes bullying.

d. If the patients are children, ask them whether they know the person that they can ask for help and support or not

e. Ask the patient if their school is involved in education about cyberbullying

f. Ask the parents whether the patients have access to education of Cyberbullying

In addition, the intervention can be done by health personnels by involving parents, among others:

a. Encourage parents to talk regularly to their children about what they do when they are online, what they like, and if there are problems that they face

b. Encourage the parents to ask children to explain or show their digital activities.

c. Encourage the parents to be supportive in responding social problems such as listening, supporting, and providing a different perspective.

d. Explain to parents that if social problems persist, they can notify the Web site or application maker about the problem. They can also tell the school, where adults can keep an eye on the interaction and support for children.

e. Encourage the parents to be creative.

4. KiVa Program

KiVa stands for (Kiusaamista Vastaan, "Fighting bullying"), it was a researchbased anti-bullying program that has been developed at Turku University, Finland, with funding from the Ministry of Education and Culture. The effectiveness of KiVa has been demonstrated in large randomized controlled trials. This study aimed to describe the health problems and evidencebased intervention programs that support trauma healing.

The principle of KiVa was the prevention of bullying by developing character in students by forming group norms. The forms were diverse, it can be videos, online games, surveys, posters, and guidebooks for parents. Children were taught a feeling of responsibility and empathy (Herkama and Salmivalli, 2017).

The KiVa program involved actions shown for students who have been involved in intimidation as bullies or victims, they refer to a series of discussions with bullies and victims with appropriate follow-up procedures.

In addition, there were universal actions (targeted at all students as witnesses of potential bullying). The program was delivered over 2 hours, with the aim of increasing awareness of peer group roles in intimidation, increasing empathy for students who were intimidated, and promoting strategies to support and strengthen the victims who were peers. Universal actions also include posters, vests for supervisors, innovative digital learning environments, antibullying computer games which can be played with a PC or tablet computer, either during or between the lessons (Poskiparta et al., 2013).

Digital KiVa games involved five levels, topics that matched the contents of 10 students' lessons. Each level included three modules: "IKnow," "ICan," and "IDo." In I Know module, students were not only learned new facts about bullying, but also test what they have learned during the lesson so far. They asked some questions about the content of lessons in the game 
and they can test themselves about various characteristics of intimidation situations (for example, Can I ignore group pressure? What is my best way to support fellow victims?).

In the I Can module, students practiced the skills they have learned. They moved in virtual schools and faced challenging situations in playgrounds, lunch rooms and school corridors. They made decisions about how to respond to this situation, and got feedback based on their choices.

At certain points of narration, players have the opportunity to "read minds" from other characters (eg victims or spectators), see how they think and feel, based on these cues, and about how the episodes take place, players can also change their behavior and try something different.

The third module, I Do, was designed to encourage students to use knowledge and skills in real life situations. This happened by asking them to report - at each level of the game - what they have practiced; for example, have they treated other people with respect, have they ignored negative pressure groups, or have they supported someone who has been intimidated. Finally, they can get feedback (Araya et al., 2017).

\section{Kiva Program Evaluation}

Since 2007, a number of randomized controlled trials have been recorded to evaluate KiV programs involving no less than 234 schools (117 interventions, 117 controls) and > 30 ooo students (Herkama and Salmivalli, 2017).

Based on the RCT study by Nocentini and Menesini (2016), which aimed to evaluate the effectiveness of the KiVa antibullying program in Italy and involved 2,042 students from 13 schools who were randomly selected and grouped for intervention (KiVa) and controls.
The results showed that KiVa which applied to students can reduce intimidation, victimization, and pro-intimidation and increase empathy to the victims. The results showed that the chances of being victimized were 1.93 times higher in the control group than in the KiVa group.

\section{DISCUSSION}

The findings of this systematic review showed the interventions that can be integrated into practical clinical guidelines and for future cyberbullying interventions.

The program with a significant reduction in cyberbullying also highlighted the importance of involving the parents, educators, and health personnels in education about cyberbullying that has been supported by Science, Engineering, and Medicine.

Various studies have also found strong evidence for parental education about cyberbullying (Ttofi and Farrington, 2011). Therefore, it can be concluded that health care providers not only provide education about cyberbullying to patients, but also to parents. In addition, research from Fenaughty and Harré (2013) found that young people were hesitant to approach their parents when they were harassed electronically, because they were afraid that their phones would be taken or blamed.

The KiVa program has been tested effectively outside Finland, and has been successfully implemented in several European countries. Therefore, if the effect of the program was positive, it was possible for a broad implementation in the future, including in Indonesia.

However, there were several challenges, which were arranging face-toface training sessions with school personnel to motivate them to implement the KiVa program as intended, and also ongoing 
support and guidance during the implementation process.

\section{REFERENCES}

Albdour M, Hong JS, Lewin L, Yarandi H (2019). The impact of cyberbullying on physical and psychological health of Arab American Adolescents. Journal of Immigrant and Minority Health. Springer.

Arseneault L. (2017). The long-term impact of bullying victimization on mental health, World Psychiatry.

Craig W, Yossi HF, Hayya FG, Suzanne D, Jorn H, Bruce SM, Michal M . et al. (2009). A cross-national profile of bullying and victimization among adolescents in 40 countries, International Journal of Public Health.

Donnerstein E, Englander E, Kowalski R, Lin CA, Parti K (2017). Defining Cyberbullying. Pediatrics, 140(2): S148-S151.

Fenaughty J, Harré N (2013). Factors associated with young people's successful resolution of distressing electronic harassmen, Computers and Education.

Van Geel M, Vedder P, Tanilon J (2014). Relationship between peer victimization, cyberbullying, and suicide in children and adolescents ametaanalysis. JAMA Pediatrics.

Gaete J, Araya R, Valenzuela D, Barohana CR, Valenzuela E, Salmivali C (2017). The KiVa antibullying program in primary schools in Chile, with and without the digital game component: study protocol for a randomized controlled trial. Crossmark. Trials 18(1): 1-9.

Gini G, Espelage DL (2014). Peer victimization, cyberbullying, and suicide risk in children and adolescents. JAMA -
Journal of the American Medical Association.

Giumetti GW, Kowalski RM (2015). Cyberbullying matters: Examining the incremental impact of cyberbullying on outcomes over and above traditional bullying in North America in Cyberbullying Across the Globe: Gender, Family, and Mental Health.

Herkama S, Salmivalli C (2017). KiVa antibullying program, Reducing Cyberbullying in Schools: International Evidence-Based Best Practices: 125134.

Hutson E, Kelly S, Militello LK (2018). Systematic review of cyberbullying interventions for youth and parents with implications for evidence-based practice. Worldviews on EvidenceBased Nursing. 15(1): 72-79.

Kowalski R (2014). Bullying in the digital age: A critical review and meta-analysis of cyberbullying research among youth". Psychological Bulletin.

Låftman SB, Modin B, Östberg V (2013). Cyberbullying and subjective health. A large-scale study of students in Stockholm, Sweden. Children and Youth Services Review.

Nocentini A, Menesini E (2016). KiVa antibullying program in Italy: Evidence of effectiveness in a randomized control trial. Prevention Science. Prevention Science, 17(8): 1012-1023.

Patchin JW, Hinduja S (2006). Bullies move beyond the schoolyard: A preliminary look at cyberbullying. Youth Violence and Juvenile Justice.

Poskiparta E, Ari K, Virpi P, Salmavalli C (2013). Bullies??? and victims??? experiences of the anti-bullying game from the KiVa program. The Impact of Technology on Relationships in Educational Settings. 
Schneider SK, Lempinen L, Brunstein KA (2012). Cyberbullying, school bullying, and psychological distress: A regional census of high school students. American Journal of Public Health.

Thomas HJ, Chan, Gary CK, Scott JG, Connor JP, Kelly AB, Williams J (2016). Association of different forms of bullying victimisation with adoles- cents' psychological distress and reduced emotional wellbeing. The Australian and New Zealand journal of psychiatry.

Ttofi MM, Farrington DP (2011). Effectiveness of school-based programs to reduce bullying: A systematic and meta-analytic review. Journal of Experimental Criminology. 